\title{
Valiron's Interpolation Formula and a Derivative Sampling Formula in the Mellin Setting Acquired via Polar-Analytic Functions
}

\author{
Carlo Bardaro ${ }^{1}$ - Paul L. Butzer ${ }^{2}$ - Ilaria Mantellini ${ }^{1} \cdot$ Gerhard Schmeisser $^{3}$ \\ Received: 11 December 2019 / Revised: 17 June 2020 / Accepted: 30 June 2020 / \\ Published online: 11 August 2020 \\ (c) The Author(s) 2020
}

\begin{abstract}
In this paper, we first recall some recent results on polar-analytic functions. Then we establish Mellin analogues of a classical interpolation of Valiron and of a derivative sampling formula. As consequences a new differentiation formula and an identity theorem in Mellin-Bernstein spaces are obtained. The main tool in the proofs is a residue theorem for polar-analytic functions.
\end{abstract}

Keywords Polar-analytic functions · Cauchy's integral formulae $\cdot$ Logarithmic poles · Exponential formula for derivatives · Mellin-Bernstein spaces · Valiron's interpolation formula $\cdot$ Identity theorem

Mathematics Subject Classification 30E20 - 30F30 $\cdot 44 \mathrm{~A} 05 \cdot 65 \mathrm{D} 25$

\section{Introduction}

The notion of polar-analytic functions was first introduced in [1] as a simple alternative for functions that are analytic on a part of the Riemann surface of the logarithm. It is of interest in the realm of Mellin analysis and the theory of quadrature formulae on the positive real axis (see $[1,2]$ ) as well as in associated computations since it avoids the

Dedicated to the memory of Professor Stephan Ruscheweyh.

Communicated by Vladimir V. Andrievskii.

Carlo Bardaro and Ilaria Mantellini have been partially supported by the "Gruppo Nazionale per l'Analisi Matematica e Applicazioni (GNAMPA)" of the "Instituto di Alta Matematica (INDAM)" as well as by the project "Ricerca di Base 2019 of University of Perugia (title: Integrazione, Approssimazione Analisi non Lineare e loro Applicazioni)".

Gerhard Schmeisser

schmeisser@mi.uni-erlangen.de

Extended author information available on the last page of the article 
necessity of specifying analytic branches. In Mellin analysis polar-analytic functions play a role similar to the one played by analytic functions in Fourier analysis. As such, polar analyticity turns out to be very helpful for the foundation of Mellin analysis independent of Fourier analysis.

First steps for a systematic development of the theory of polar-analytic functions were made in the recent paper [3], in which an analogue of Cauchy's integral formula and Taylor-type series were established. However, the integral formula given in [3] is useful in horizontal strips of width less than $2 \pi$ only since otherwise it may produce additional residues as undesired artifacts. Therefore in [4] a further analogue of Cauchy's integral formula was achieved, which is always free of artifacts. It allowed us to deduce an efficient residue theorem for polar-analytic functions. Based on this theorem, we established an analogue of Boas' differentiation formula for polar Mellin derivatives and, as a consequence, a Bernstein type inequality for polar Mellin derivatives.

In the present paper, as a further application of our theory of polar-analytic functions, we employ the new residue theorem of [4] for deducing Mellin analogues of Valiron's interpolation formula and of a derivative sampling formula. We mention that an abstract approach to Valiron's formula as well as to Boas' differentiation formula was studied by I. Pesenson (see [13, Thm. 4.1] and [14, Thm. 3.3]) in the general setting of groups of operators in Banach spaces (see [6]). Indeed, the Mellin derivative used here can be viewed as the infinitesimal generator of the group of operators generated by the Mellin translation operator on the Banach space $X_{c}^{p}$ (see Sect. 4), defined by

$$
T(t) f(r):=t^{c} f(t r), \quad t \in \mathbb{R}^{+}, f \in X_{c}^{p} .
$$

The theory of polar-analytic functions enables us to obtain a direct approach to sampling formulae in the Mellin setting, fully different from that considered in [13,14].

The paper is organized as follows. In Sect. 2, we introduce the notion of polar analyticity and present some formulae for Mellin derivatives (the Mellin analogue of ordinary derivatives) and polar Mellin derivatives (the Mellin analogue of complex derivatives). In Sect. 3, we present some basic results on polar-analytic functions, among them being the residue theorem from [4]. In Sect. 4, we recall Mellin-Bernstein spaces, a Mellin version of the Paley-Wiener theorem and the aforementioned analogue of Boas' differentiation formula as well as a consequence of it. As a new result of this paper, a Mellin analogue of Valiron's interpolation formula is established in Sect. 5. It gives access to a new differentiation formula which has some advantages over that of Boas. Furthermore, it leads to an identity theorem in Mellin-Bernstein spaces. In Sect. 6, we use the Mellin version of the Paley-Wiener theorem and the residue theorem for polar-analytic functions for acquiring a sampling formulae for Mellin-bandlimited functions which involves also samples of Mellin derivatives up to a given order $m$. Finally, in Sect. 7 we turn to computational aspects by estimating the truncation errors for three of the previous formulae.

We wish to dedicate the present article to the memory of the late Professor Stephan Ruscheweyh in appreciation of his excellent achievements in mathematical research and education. One of us, Gerhard Schmeisser, felt particularly close to him and adds a few personal words: Although our universities in Erlangen and Würzburg are 
located within a distance of around hundred kilometers only, it was via Montreal that a close relationship and friendship was built up between us. We were both guests of Q.I. Rahman and lived in his house in Outremont (Montreal) in the early eighties of the last century. From that time on, Stephan R. counted me as one of the persons in his scientific field and invited me to the various conferences promoted or organized by him, such as the CMFT conferences, Tag der Funktionentheorie, Oberwolfach meetings and the Bavaria-Quebec meetings. He also gave a lecture on the occasion of my 65 th birthday anniversary and my retirement in June 2004. I reciprocated a little by refereeing numerous manuscripts which he handled as an editor of several journals. When I sought his advice, he was always willing to communicate with me in detailed emails or long telephone calls. Among his many talents, I admired his sharp and quick mind as well as his skills as an organizer. I miss him very much.

\section{Preliminaries}

Throughout this paper, we denote by $\mathbb{R}^{+}$the set of positive real numbers. Let $\mathbb{H}:=$ $\left\{(r, \theta) \in \mathbb{R}^{+} \times \mathbb{R}\right\}$ be the right half-plane and let $\mathcal{D}$ be a domain in $\mathbb{H}$, which means that $\mathcal{D}$ is open, connected and non-empty. The following notion will be of fundamental importance in our considerations.

Definition 1 We say that $f: \mathcal{D} \rightarrow \mathbb{C}$ is polar-analytic on $\mathcal{D}$ with polar derivative $D_{\text {pol }} f$ if for any $\left(r_{0}, \theta_{0}\right) \in \mathcal{D}$ the limit

$$
\lim _{(r, \theta) \rightarrow\left(r_{0}, \theta_{0}\right)} \frac{f(r, \theta)-f\left(r_{0}, \theta_{0}\right)}{r e^{i \theta}-r_{0} e^{i \theta_{0}}}=:\left(D_{\mathrm{pol}} f\right)\left(r_{0}, \theta_{0}\right)
$$

exists and is the same howsoever $(r, \theta)$ approaches $\left(r_{0}, \theta_{0}\right)$ within $\mathcal{D}$.

The theory of polar-analytic functions was outlined in [3]. As basic facts, polar analyticity of two functions is inherited by their arithmetic combinations and the familiar rules known for classical differentiation hold for polar derivatives as well. Moreover, for a polar-analytic function $f:(r, \theta) \mapsto u(r, \theta)+i v(r, \theta)$ on $\mathcal{D}$, where $u$ and $v$ are real-valued, we may interpret $f$ as a mapping from a subset of the halfplane $\mathbb{H}$ into $\mathbb{R}^{2}$, and then it turns out that $f$ is differentiable in the classical sense of real analysis. Finally, it can be verified that $f=u+i v$, where $u, v: \mathcal{D} \rightarrow \mathbb{R}$, is polar-analytic on $\mathcal{D}$ if and only if $u$ and $v$ have continuous partial derivatives on $\mathcal{D}$ that satisfy the differential equations

$$
\frac{\partial u}{\partial \theta}=-r \frac{\partial v}{\partial r}, \quad \frac{\partial v}{\partial \theta}=r \frac{\partial u}{\partial r} .
$$

Note that these equations coincide with the Cauchy-Riemann equations of an analytic function $g$ defined by $g(z):=u(r, \theta)+i v(r, \theta)$ for $z=r e^{i \theta}$; see, e.g., [5, Sect. 23, p. 68], [12, Sect. 4.3 , pp. 78-82] or [15]. For the derivative $D_{\text {pol }}$, we easily find that

$$
\left(D_{\mathrm{pol}} f\right)(r, \theta)=e^{-i \theta}\left(\frac{\partial}{\partial r} u(r, \theta)+i \frac{\partial}{\partial r} v(r, \theta)\right)
$$




$$
=\frac{e^{-i \theta}}{r}\left(\frac{\partial}{\partial \theta} v(r, \theta)-i \frac{\partial}{\partial \theta} u(r, \theta)\right) .
$$

Since $f=u+i v$, equations (1) can be written in a more compact way as

$$
\frac{\partial f}{\partial \theta}=i r \frac{\partial f}{\partial r}
$$

and then formula (2) takes the form

$$
\left(D_{\mathrm{pol}} f\right)(r, \theta)=e^{-i \theta} \frac{\partial}{\partial r} f(r, \theta)=\frac{e^{-i \theta}}{i r} \frac{\partial}{\partial \theta} f(r, \theta) .
$$

Also note that $D_{\text {pol }}$ is the ordinary differentiation on $\mathbb{R}^{+}$. More precisely, if $\varphi(\cdot):=$ $f(\cdot, 0)$, then $\left(D_{\text {pol }} f\right)(r, 0)=\varphi^{\prime}(r)$.

As to connections with the classical theory of analytic functions, when $g$ is an entire function, then $f:(r, \theta) \mapsto g\left(r e^{i \theta}\right)$ defines a function $f$ on $\mathbb{H}$ that is polaranalytic and $2 \pi$-periodic with respect to $\theta$, and one has $\left(D_{\text {pol }} f\right)(r, \theta)=g^{\prime}(z)$ with $z=r e^{i \theta}$. A converse statement is not true in general. If $f$ is polar-analytic on $\mathbb{H}$ and $2 \pi$-periodic with respect to the second variable, there need not exist an entire function $h$ such that $f(r, \theta)=h\left(r e^{i \theta}\right)$. A simple example is the function $f(r, \theta):=e^{-i \theta} / r$. It would imply that $h(z)=1 / z$, which is analytic on $\mathbb{C} \backslash\{0\}$ only. However, if $f$ is a polar-analytic function on $\mathbb{H}$, then $g: z=x+i y \mapsto f\left(e^{x}, y\right)$ is an entire function.

The main novelty of the definition of polar-analytic function is that, using this approach, we avoid periodicity with respect to the $\operatorname{argument} \theta$, and in this way we can avoid the use of Riemann surfaces.

A simple example of a polar-analytic function that is not $2 \pi$-periodic is the function $L(r, \theta):=\log r+i \theta$, which is easily seen to satisfy the differential equations (1). In this way, the logarithm is described as a single-valued function on $\mathbb{H}$, without using its Riemann surface $S_{\log }$. Moreover, with $z=r e^{i \theta}$, we find that

$$
\left(D_{\mathrm{pol}} L\right)(r, \theta)=e^{-i \theta} \frac{1}{r}=\frac{1}{r e^{i \theta}}=\frac{1}{z} .
$$

Next we recall the definition of the (pointwise) Mellin derivative of a function $f: \mathbb{R}^{+} \rightarrow \mathbb{C}$; see [7]. For $c \in \mathbb{R}$, it is denoted by $\Theta_{c} f$ and defined as

$$
\Theta_{c} f(x):=x f^{\prime}(x)+c f(x), \quad x \in \mathbb{R}^{+},
$$

provided that $f^{\prime}$ exists a.e. on $\mathbb{R}^{+}$. It is extended to order $k \in \mathbb{N}$ by defining recursively $\Theta_{c}^{1}:=\Theta_{c}, \Theta_{c}^{k}:=\Theta_{c}\left(\Theta_{c}^{k-1}\right)$. For convenience, we set $\Theta^{k}:=\Theta_{0}^{k}$ and $\Theta_{c}^{0}:=I$ with $I$ denoting the identity operator.

The Mellin derivative of order $k \in \mathbb{N}$ has the representation

$$
\Theta_{c}^{k} f(x)=\sum_{j=0}^{k} S_{c}(k, j) x^{j} f^{(j)}(x)
$$


where $S_{c}(k, j)$ are the generalized Stirling number of the second kind; see [7].

For $c \in \mathbb{R}$, we define the polar Mellin derivative of a polar-analytic function $f$ by the formula

$$
\widetilde{\Theta}_{c} f(r, \theta):=r e^{i \theta}\left(D_{\mathrm{pol}} f\right)(r, \theta)+c f(r, \theta) .
$$

As before, for $\varphi(r):=f(r, 0)$, we have $\widetilde{\Theta}_{c} f(r, 0)=\left(\Theta_{c} \varphi\right)(r)$, where $\Theta_{c} f$ is the (usual) Mellin derivative.

The higher order polar Mellin derivatives may be defined through the representation formula for (usual) Mellin derivatives (4), namely

$$
\widetilde{\Theta}_{c}^{k} f(r, \theta):=\sum_{j=0}^{k} S_{c}(k, j) r^{j} e^{i j \theta} D_{\text {pol }}^{j} f(r, \theta), \quad k \in \mathbb{N},(r, \theta) \in \mathbb{H} .
$$

\section{Some Basic Results on Polar-Analytic Functions}

We recall some basic results from the theory of polar-analytic functions which will be useful in the present paper; see [4] for proofs and more details.

For $\left(r_{0}, \theta_{0}\right) \in \mathbb{H}$ and $\rho>0$, the polar disk centered at $\left(r_{0}, \theta_{0}\right)$ with radius $\rho$, is defined by

$$
E\left(\left(r_{0}, \theta_{0}\right), \rho\right):=\left\{(r, \theta) \in \mathbb{H}:\left(\log \frac{r}{r_{0}}\right)^{2}+\left(\theta-\theta_{0}\right)^{2}<\rho^{2}\right\}
$$

Theorem 1 Let $f: \mathcal{D} \rightarrow \mathbb{C}$ be polar-analytic on a domain $\mathcal{D} \subset \mathbb{H}$ and let $c \in \mathbb{R}$. If $\left(r_{0}, \theta_{0}\right) \in \mathcal{D}$, then there holds the expansion

$$
\left(r e^{i \theta}\right)^{c} f(r, \theta)=\left(r_{0} e^{i \theta_{0}}\right)^{c} \sum_{k=0}^{\infty} \frac{\left(\widetilde{\Theta}_{c}^{k} f\right)\left(r_{0}, \theta_{0}\right)}{k !}\left(\log \frac{r}{r_{0}}+i\left(\theta-\theta_{0}\right)\right)^{k}
$$

converging uniformly on every polar disk $E\left(\left(r_{0}, \theta_{0}\right), \rho\right) \subset \mathcal{D}$.

As a consequence of the series expansion (7), we deduced in [4] the following identity theorem for polar-analytic functions.

Theorem 2 Let $\mathcal{D}$ be a domain in $\mathbb{H}$ and let $f: \mathcal{D} \rightarrow \mathbb{C}$ be polar-analytic. Suppose that $\left(r_{0}, \theta_{0}\right) \in \mathcal{D}$ is an accumulation point of distinct zeros of $f$. Then $f$ is identically zero.

In the following, a curve will be called regular if it is piecewise continuously differentiable. The Cauchy integral formula for polar-analytic functions can now be stated as follows; see [4] for a proof.

Theorem 3 Let $\mathcal{D}$ be a convex domain in $\mathbb{H}$, and let $f: \mathcal{D} \rightarrow \mathbb{C}$ be polar-analytic on $\mathcal{D}$. Let $\gamma$ be a positively oriented, closed, regular curve that is the boundary of a 
convex domain $\operatorname{int}(\gamma) \subset \mathcal{D}$. Then, for $\left(r_{0}, \theta_{0}\right) \in \operatorname{int}(\gamma), c \in \mathbb{R}$ and $k \in \mathbb{N}_{0}$, we have

$$
\frac{1}{2 \pi i} \int_{\gamma} \frac{\left(r e^{i \theta}\right)^{c-1} f(r, \theta) e^{i \theta}}{\left(\log \left(\frac{r}{r_{0}}\right)+i\left(\theta-\theta_{0}\right)\right)^{k+1}}(d r+i r d \theta)=\left(r_{0} e^{i \theta_{0}}\right)^{c} \frac{\left(\widetilde{\Theta}_{c}^{k} f\right)\left(r_{0}, \theta_{0}\right)}{k !}
$$

The basic tool for the new results in this paper is the subsequent residue theorem for polar-analytic functions. For its statement, we first recall some notions; see [4].

Definition 2 Let $\left(r_{0}, \theta_{0}\right) \in \mathbb{H}$ and let $\mathcal{U} \subset \mathbb{H}$ be an open neighborhood of $\left(r_{0}, \theta_{0}\right)$.

(i) If $f: \mathcal{U} \backslash\left\{\left(r_{0}, \theta_{0}\right)\right\} \rightarrow \mathbb{C}$ is polar-analytic, then $\left(r_{0}, \theta_{0}\right)$ will be called an isolated singularity of $f$.

(ii) An isolated singularity $\left(r_{0}, \theta_{0}\right)$ is said to be a logarithmic pole of order $k$ if $k \in \mathbb{N}$ and there exists a polar-analytic function $g: \mathcal{U} \rightarrow \mathbb{C}$ with $g\left(r_{0}, \theta_{0}\right) \neq 0$ such that

$$
f(r, \theta)=\frac{g(r, \theta)}{\left(\log \left(\frac{r}{r_{0}}\right)+i\left(\theta-\theta_{0}\right)\right)^{k}} \quad \text { for }(r, \theta) \in \mathcal{U} \backslash\left\{\left(r_{0}, \theta_{0}\right)\right\} .
$$

In this case

$$
\left(\operatorname{res}_{c} f\right)\left(r_{0}, \theta_{0}\right):=\left(r_{0} e^{i \theta_{0}}\right)^{c} \frac{\left(\widetilde{\Theta}_{c}^{k-1} g\right)\left(r_{0}, \theta_{0}\right)}{(k-1) !}
$$

will be called the $c$-residue of $f$ at $\left(r_{0}, \theta_{0}\right)$.

Now the residue theorem for logarithmic poles can be stated as follows; see [4] for a proof.

Theorem 4 Let $\mathcal{D}$ be a convex domain in $\mathbb{H}$, and let $f$ be polar-analytic on $\mathcal{D}$ except for isolated singularities which are all logarithmic poles. Let $\gamma$ be a positively oriented, closed, regular curve that is the boundary of a convex domain $\operatorname{int}(\gamma) \subset \mathcal{D}$. Suppose that no isolated singularity lies on $\gamma$ while $\left(r_{j}, \theta_{j}\right)$ for $j=1, \ldots, m$ are the isolated singularities lying in $\operatorname{int}(\gamma)$. Then, for $c \in \mathbb{R}$, there holds

$$
\int_{\gamma}\left(r e^{i \theta}\right)^{c-1} f(r, \theta) e^{i \theta}(d r+i r d \theta)=2 \pi i \sum_{j=1}^{m}\left(\operatorname{res}_{c} f\right)\left(r_{j}, \theta_{j}\right) .
$$

Remark 1 Let $f$ be polar-analytic in a neighborhood of a point $(r, 0) \in \mathbb{H}$. Then it can be verified that

$$
\left(\widetilde{\Theta}_{c}^{k} f\right)(r, 0)=\left(\Theta_{c}^{k} f(\cdot, 0)\right)(r) .
$$

This simplifies the calculation of $c$-residues at points in $\mathbb{R}^{+}$. 
Remark 2 The proof of [4, Thm. 4] reveals that, if

$$
f(r, \theta)=\frac{g(r, \theta)}{\left(\log \left(\frac{r}{r_{0}}\right)+i\left(\theta-\theta_{0}\right)\right)^{k}}=\frac{h(r, \theta)}{\left(\log \left(\frac{r}{r_{0}}\right)+i\left(\theta-\theta_{0}\right)\right)^{k-j}}
$$

with $j \in \mathbb{N}, h\left(r_{0}, \theta_{0}\right) \neq 0$ and (consequently) $g\left(r_{0}, \theta_{0}\right)=0$, then

$$
\left(\operatorname{res}_{c} f\right)\left(r_{0}, \theta_{0}\right):=\left(r_{0} e^{i \theta_{0}}\right)^{c} \frac{\left(\widetilde{\Theta}_{c}^{k-j-1} h\right)\left(r_{0}, \theta_{0}\right)}{(k-j-1) !}=\left(r_{0} e^{i \theta_{0}}\right)^{c} \frac{\left(\widetilde{\Theta}_{c}^{k-1} g\right)\left(r_{0}, \theta_{0}\right)}{(k-1) !}
$$

In other words, if $f$ has a logarithmic pole of order at most $k$, we may naively use the formula for a logarithmic pole of order $k$ for calculating the $c$-residue.

\section{Mellin-Bernstein Spaces and Boas' Differentiation Formula for Polar Mellin Derivatives}

For $p \in\left[1, \infty\left[\right.\right.$, denote by $\|\cdot\|_{p}$ the norm of the Lebesgue space $L^{p}\left(\mathbb{R}^{+}\right)$. In Mellin analysis, the analogue of $L^{p}\left(\mathbb{R}^{+}\right)$are the spaces $X_{c}^{p}$, where $c \in \mathbb{R}$, comprising all functions $f: \mathbb{R}^{+} \rightarrow \mathbb{C}$ such that $f(\cdot)(\cdot)^{c-1 / p} \in L^{p}\left(\mathbb{R}^{+}\right)$with the norm $\|f\|_{X_{c}^{p}}:=\left\|f(\cdot)(\cdot)^{c-1 / p}\right\|_{p}$. Furthermore, for $p=\infty$, we define $X_{c}^{\infty}$ as the space of all measurable functions $f: \mathbb{R}^{+} \rightarrow \mathbb{C}$ such that $\|f\|_{X_{c}^{\infty}}:=\sup _{x>0} x^{c}|f(x)|<\infty$; see [7].

We recall (see [1]) that for $T>0$ the Mellin-Bernstein space $\mathscr{B}_{c, T}^{p}$ comprises all functions $f: \mathbb{H} \rightarrow \mathbb{C}$ with the following properties:

(i) $f$ is polar-analytic on $\mathbb{H}$;

(ii) $f(\cdot, 0) \in X_{c}^{p}$;

(iii) there exists a constant $C_{f}>0$ such that $r^{c}|f(r, \theta)| \leq C_{f} e^{T|\theta|}$ for all $(r, \theta) \in \mathbb{H}$.

A relevant property of the Mellin-Bernstein spaces is the Mellin version of the Paley-Wiener theorem for $p=2$, given in [1, Thm. 5.1]. To recall it, we may proceed as follows: For $f \in X_{c}^{2}$, denote by $[f]_{M_{c}^{2}}^{\wedge}$ the Mellin transform of $f$ (see $[7,9]$ ). Then the Paley-Wiener space $B_{c, T}^{2}$ comprises all functions $f \in X_{c}^{2} \cap C\left(\mathbb{R}^{+}\right)$such that $[f]_{M_{c}^{2}}^{\wedge}(c+i t)=0$ a.e. for $|t|>T$. These functions are also called Mellin-bandlimited to the interval $[-T, T]$; see $[9, \S 5.1]$.

Now the polar-analytic Mellin version of the Paley-Wiener theorem in $X_{c}^{2}$-spaces affirms that a function $\varphi \in X_{c}^{2}$ belongs to the Paley-Wiener space $B_{c, T}^{2}$ if and only if there exists a function $f \in \mathscr{B}_{c, T}^{2}$ such that $f(\cdot, 0)=\varphi(\cdot)$.

Next we state three useful assertions on transformations in Mellin-Bernstein spaces. They are verified by straightforward calculations. To show polar analyticity, we simply check that the Cauchy-Riemann equations in polar form are satisfied. The first statement affirms that $\mathscr{B}_{c, T}^{p}$ is invariant under Mellin translations. 
Proposition 1 Let $f \in \mathscr{B}_{c, T}^{p}$, where $p \geq 1, c \in \mathbb{R}$ and $T>0$. For $t>0$, define

$$
g:(r, \theta) \longmapsto t^{c} f(t r, \theta) .
$$

Then $g \in \mathscr{B}_{c, T}^{p}$; in particular, $\|g(\cdot, 0)\|_{X_{c}^{p}}=\|f(\cdot, 0)\|_{X_{c}^{p}}$ and $r^{c}|g(r, \theta)| \leq C_{f} e^{T|\theta|}$ for all $(r, \theta) \in \mathbb{H}$. Furthermore,

$$
\left(\widetilde{\Theta}_{c} g\right)(r, \theta)=t^{c}\left(\widetilde{\Theta}_{c} f\right)(t r, \theta) .
$$

The second statement concerns a transformation of $T$.

Proposition 2 Let $f \in \mathscr{B}_{c, T}^{p}$, where $p \geq 1, c \in \mathbb{R}$ and $T>0$. Define

$$
h:(r, \theta) \longmapsto f\left(r^{1 / T}, \frac{\theta}{T}\right) .
$$

Then $h \in \mathscr{B}_{c / T, 1}^{p}$; in particular, $\|h(\cdot, 0)\|_{X_{c / T}^{p}}=\|f(\cdot, 0)\|_{X_{c}^{p}}$ and $r^{c / T}|h(r, \theta)| \leq$ $C_{f} e^{|\theta|}$ for all $(r, \theta) \in \mathbb{H}$. Furthermore,

$$
\left(\widetilde{\Theta}_{c / T} h\right)(r, \theta)=\frac{1}{T}\left(\widetilde{\Theta}_{c} f\right)\left(r^{1 / T}, \frac{\theta}{T}\right) .
$$

The third statement concerns a shift of the second argument of $f$. For verifying property (ii) in the definition of Mellin-Bernstein spaces, one uses [1, Thm. 4.2].

Proposition 3 Let $f \in \mathscr{B}_{c, T}^{p}$, where $p \geq 1, c \in \mathbb{R}$ and $T>0$. For $\alpha \in \mathbb{R}$, define

$$
\phi:(r, \theta) \longmapsto f(r, \theta+\alpha) .
$$

Then $\phi \in \mathscr{B}_{c, T}^{p}$; in particular, $\|\phi(\cdot, 0)\|_{X_{c}^{p}} \leq e^{T|\alpha|}\|f(\cdot, 0)\|_{X_{c}^{p}}$ and $r^{c}|\phi(r, \theta)| \leq$ $C_{f} e^{T(|\alpha|+|\theta|)}$ for all $(r, \theta) \in \mathbb{H}$. Furthermore,

$$
\left(\widetilde{\Theta}_{c} \phi\right)(r, \theta)=\left(\widetilde{\Theta}_{c} f\right)(r, \theta+\alpha)
$$

Now we present a formula for the polar Mellin derivatives. It is the Mellin version of a classical differentiation formula by Boas for bandlimited functions of Fourier analysis. For a proof see [4].

Theorem 5 Let $f \in \mathscr{B}_{c, T}^{p}$, where $p \geq 1, c \in \mathbb{R}$ and $T>0$. Then

$$
\left(\widetilde{\Theta}_{c} f\right)(r, \theta)=\frac{4 T}{\pi^{2}} \sum_{k \in \mathbb{Z}} \frac{(-1)^{k}}{(2 k+1)^{2}} e^{(k+1 / 2) \pi c / T} f\left(r e^{(k+1 / 2) \pi / T}, \theta\right)
$$

for $(r, \theta) \in \mathbb{H}$. Multiplied by $r^{c}$, the series converges absolutely and uniformly on strips of bounded width parallel to the ray $\{(r, \theta) \in \mathbb{H}: r>0, \theta=0\}$. 
As a consequence of Theorem 5, we can state the following Bernstein inequality for polar Mellin derivatives.

Corollary 1 Let $f \in \mathscr{B}_{c, T}^{p}$, where $p \in[1,+\infty], c \in \mathbb{R}$ and $T>0$. Then

$$
\left\|\left(\widetilde{\Theta}_{c} f\right)(\cdot, \theta)\right\|_{X_{c}^{p}} \leq T\|f(\cdot, \theta)\|_{X_{c}^{p}}
$$

for any $\theta \in \mathbb{R}$.

Knowing from Theorem 3 that the polar Mellin derivative of a polar-analytic function is again polar-analytic, we can also deduce from Theorem 5 the following statement (for details see [4]):

Corollary 2 The Mellin-Bernstein spaces are invariant under polar Mellin differentiation, that is, if $f \in \mathscr{B}_{c, T}^{p}$, where $p \in[1,+\infty], c \in \mathbb{R}$ and $T>0$, then $\widetilde{\Theta}_{c} f \in \mathscr{B}_{c, T}^{p}$.

Combining Corollaries 1 and 2, we can apply (10) repeatedly and obtain

$$
\left\|\left(\widetilde{\Theta}_{c}^{k} f\right)(\cdot, \theta)\right\|_{X_{c}^{p}} \leq T^{k}\|f(\cdot, \theta)\|_{X_{c}^{p}}, \quad \theta \in \mathbb{R}, k \in \mathbb{N} .
$$

\section{A Mellin Analogue of Valiron's Interpolation Formula}

In Fourier analysis, there exists an interpolation formula (or sampling formula) of Valiron-sometimes also attributed to Tschakaloff (see e.g. [11, p. 60], [10]) — that improves upon the classical Whittaker-Kotel'nikov-Shannon sampling formula. It applies to a wider class of functions and its series converges faster. The only price one has to pay for this improvement is that, apart from the samples of the classical sampling formula, also the value of the derivative at zero is needed.

For the subsequent considerations, we recall the sinc function, defined by

$$
\operatorname{sinc} z:=\left\{\begin{array}{cl}
\frac{\sin (\pi z)}{\pi z} & \text { if } z \in \mathbb{C} \backslash\{0\} \\
1 & \text { if } z=0
\end{array}\right.
$$

Here we want to establish an analogue of Valiron's formula in the Mellin setting. Our approach is similar to the derivation of the analogue of Boas' differentiation formula (Theorem 5) in [4].

Theorem 6 Let $f \in \mathscr{B}_{c, T}^{\infty}$, where $c \in \mathbb{R}$ and $T \in \mathbb{R}^{+}$. Then, for $r \in \mathbb{R}^{+}$,

$$
\begin{aligned}
r^{c} f(r, 0)= & \sin (T \log r)\left[\frac{\left(\widetilde{\Theta}_{c} f\right)(1,0)}{T}+\frac{f(1,0)}{T \log r}\right. \\
& \left.+T \log r \sum_{k \in \mathbb{Z} \backslash\{0\}} \frac{(-1)^{k+1} e^{k \pi c / T} f\left(e^{k \pi / T}, 0\right)}{k \pi(k \pi-T \log r)}\right] .
\end{aligned}
$$


The series converges absolutely and uniformly on compact subsets of $\mathbb{R}^{+}$.

Proof First we suppose that $T=1$. Let $t \in \mathbb{R}^{+} \backslash\left\{e^{k \pi}: k \in \mathbb{Z}\right\}$ be arbitrary but fixed and consider the function

$$
F:(r, \theta) \longmapsto \frac{f(r, \theta)}{\left(\log \left(\frac{r}{t}\right)+i \theta\right)(\log r+i \theta) \sin (\log r+i \theta)} .
$$

It is polar-analytic on $\mathbb{H}$ except for isolated singularities at the points where the denominator vanishes. These points are $(t, 0)$ and $\left(e^{k \pi}, 0\right)$ for all $k \in \mathbb{Z}$. The point $(1,0)$, obtained for $k=0$, is a logarithmic pole of order at most two while all other isolated singularities are logarithmic poles of order at most one.

Let $n \in \mathbb{N}$ be such that $e^{-n \pi}<t<e^{n \pi}$, and denote by $\partial \mathcal{R}_{n}$ the positively oriented rectangle with vertices at the points $\left(e^{ \pm(n+1 / 2) \pi}, \pm(n+1 / 2) \pi\right)$. By Theorem 4 , we have

$$
\frac{1}{2 \pi i} \int_{\partial \mathcal{R}_{n}}\left(r e^{i \theta}\right)^{c-1} F(r, \theta) e^{i \theta}(d r+i r d \theta)=\left(\operatorname{res}_{c} F\right)(t, 0)+\sum_{k=-n}^{n}\left(\operatorname{res}_{c} F\right)\left(e^{k \pi}, 0\right)
$$

First we want to show that the integral on the left-hand side approaches zero as $n \rightarrow \infty$. Since $f$ was assumed to belong to $\mathscr{B}_{c, 1}^{\infty}$, we have

$$
|f(r, \theta)| \leq C_{f} r^{-c} e^{|\theta|}
$$

Furthermore, it can be verified that on $\partial \mathcal{R}_{n}$

$$
|\sin (\log r+i \theta)| \geq \frac{e^{|\theta|}}{3}
$$

Thus

$$
\left|r^{c-1} F(r, \theta)\right| \leq \frac{3 C_{f}}{r\left(\log ^{2}\left(\frac{r}{t}\right)+\theta^{2}\right)^{1 / 2}\left(\log ^{2} r+\theta^{2}\right)^{1 / 2}} .
$$

For an estimate of the contributions coming from the horizontal parts of $\partial \mathcal{R}_{n}$, we have to substitute $\theta= \pm(n+1 / 2) \pi$ in (12). Replacing in addition the squared logarithms by zero, we obtain for the integrals under considerations the upper bound

$$
\frac{3}{\pi} C_{f} \int_{e^{-(n+1 / 2) \pi}}^{e^{(n+1 / 2) \pi}} \frac{1}{\left(n+\frac{1}{2}\right)^{2} \pi^{2}} \frac{d r}{r}
$$

which approaches zero as $n \rightarrow \infty$. For the contributions coming from the vertical parts, we have to substitute $r=e^{ \pm(n+1 / 2) \pi}$ in (12). Replacing in addition $\theta^{2}$ by zero, we obtain an upper bound for the corresponding integrals which again approaches zero as $n \rightarrow \infty$. Hence the above residue formula yields

$$
\left(\operatorname{res}_{c} F\right)(t, 0)=-\sum_{k \in \mathbb{Z}}\left(\operatorname{res}_{c} F\right)\left(e^{k \pi}, 0\right) .
$$


Next we calculate the residues. It is easily seen that

$$
\left(\operatorname{res}_{c} F\right)(t, 0)=\frac{t^{c} f(t, 0)}{\log t \sin (\log t)}
$$

For calculating the residues on the right-hand side of (13), we factor the sine with the help of the sinc function as

$$
\sin z=(-1)^{k}(z-k \pi) \operatorname{sinc}\left(\frac{z}{\pi}-k\right), \quad z \in \mathbb{C}, k \in \mathbb{Z}
$$

Then

$$
\left(\operatorname{res}_{c} F\right)\left(e^{k \pi}, 0\right)=(-1)^{k} \frac{e^{c k \pi} f\left(e^{k \pi}, 0\right)}{k \pi(k \pi-\log t)}, \quad k \neq 0 .
$$

Since in a neighborhood of $(1,0)$ we may write

$$
F(r, \theta)=\frac{1}{(\log r+i \theta)^{2}} \frac{f(r, \theta)}{\left(\log \left(\frac{r}{t}\right)+i \theta\right) \operatorname{sinc}\left(\frac{\log r+i \theta}{\pi}\right)}=: \frac{g(r, \theta)}{(\log r+i \theta)^{2}},
$$

we obtain

$$
\left(\operatorname{res}_{c} F\right)(1,0)=\left(\widetilde{\Theta}_{c} g\right)(1,0)=\left(D_{\mathrm{pol}} g\right)(1,0)+c g(1,0) .
$$

Now, after a calculation of the polar derivative of $g$, we arrive at

$$
\left(\operatorname{res}_{c} F\right)(1,0)=-\left[\frac{\left(\widetilde{\Theta}_{c} f\right)(1,0)}{\log t}+\frac{f(1,0)}{\log ^{2} t}\right] .
$$

With these values for the residues, formula (13) may be rewritten as

$t^{c} f(t, 0)=\sin (\log t)\left[\left(\widetilde{\Theta}_{c} f\right)(1,0)+\frac{f(1,0)}{\log t}+\log t \sum_{k \in \mathbb{Z} \backslash\{0\}} \frac{(-1)^{k+1} e^{c k \pi} f\left(e^{k \pi}, 0\right)}{k \pi(k \pi-\log t)}\right]$.

This formula holds for $f \in \mathscr{B}_{c, 1}^{\infty}$ and all $t \in \mathbb{R}^{+}$, even at the points $t=e^{k \pi}$ since

$$
\frac{\sin (\log t)}{k \pi-\log t}=(-1)^{k+1} \operatorname{sinc}\left(\frac{\log t}{\pi}-k\right)
$$

has a continuous continuation to the whole of $\mathbb{R}^{+}$.

With the help of Proposition 2, the achieved formula can be extended from $T=1$ to arbitrary $T \in \mathbb{R}^{+}$. Then, substituting $t^{1 / T}=r$, we obtain the formula stated in Theorem 6.

Noting that $e^{k \pi c / T}\left|f\left(e^{k \pi / T}, 0\right)\right| \leq C_{f}$ for all $k \in \mathbb{Z}$, we easily verify the assertion on the convergence of the series. 
Remark 3 In order to avoid undetermined expressions that need continuation, we may write the formula of Theorem 6 in terms of lin functions as introduced in [8]. For $c \in \mathbb{R}$ and $x \in \mathbb{R}^{+}$, they are defined by

$$
\operatorname{lin}_{c}(x):=x^{-c} \operatorname{sinc}(\log x)
$$

with the continuous extension $\operatorname{lin}_{c}(1):=1$. Indeed, we have

$$
\begin{aligned}
f(r, 0)= & \operatorname{lin}_{c \pi / T}\left(r^{T / \pi}\right)\left[\log r\left(\widetilde{\Theta}_{c} f\right)(1,0)+f(1,0)\right] \\
& +\log \left(r^{T / \pi}\right) \sum_{k \in \mathbb{Z} \backslash\{0\}} \frac{f\left(e^{k \pi / T}, 0\right)}{k} \operatorname{lin}_{c \pi / T}\left(e^{-k} r^{T / \pi}\right) .
\end{aligned}
$$

This formula compares well with the exponential sampling formula in [8, Thm. 6.3] and its right-hand side is defined for all $r \in \mathbb{R}^{+}$. On the other hand, it is very inconvenient when we want to estimate $f$ using property (iii) of the Mellin-Bernstein space since all factors depending on $c$ are hidden in the lin functions. Another way to write the above formula is

$$
\begin{aligned}
r^{c} f(r, 0)= & \operatorname{lin}_{0}\left(r^{T / \pi}\right)\left[\log r\left(\widetilde{\Theta}_{c} f\right)(1,0)+f(1,0)\right] \\
& +\log \left(r^{T / \pi}\right) \sum_{k \in \mathbb{Z} \backslash\{0\}} \frac{e^{k \pi c / T} f\left(e^{k \pi / T}, 0\right)}{k} \operatorname{lin}_{0}\left(e^{-k} r^{T / \pi}\right) .
\end{aligned}
$$

Now the factors depending on $c$ can go with $f$ as needed in (iii).

Valiron's formula and its Mellin analogue will serve primarily for the reconstruction of a function $f$ from samples. Among the latter, there is also a sample of the derivative of $f$. One may therefore think of interpreting this formula as a representation of that derivative of $f$ by samples of $f$. In the following corollary, we establish such a differentiation formula by skillful use of Theorem 6 . As we shall see, it has some advantages over the corresponding analogue of Boas' differentiation formula. It seems that even a Fourier version of this result has not yet been explicitly mentioned in the literature.

Corollary 3 Let $f \in \mathscr{B}_{c, T}^{\infty}$, where $c \in \mathbb{R}$ and $T>0$. Then

$$
\begin{aligned}
\left(\widetilde{\Theta}_{c} f\right)(r, \theta)= & \frac{T}{2}\left[e^{\pi c /(2 T)} f\left(r e^{\pi /(2 T)}, \theta\right)-e^{-\pi c /(2 T)} f\left(r e^{-\pi /(2 T)}, \theta\right)\right] \\
& +\frac{T}{\pi} \sum_{k \in \mathbb{Z} \backslash\{0\}} \frac{(-1)^{k} e^{k \pi c / T} f\left(r e^{k \pi / T}, \theta\right)}{k\left(4 k^{2}-1\right)}
\end{aligned}
$$

for $(r, \theta) \in \mathbb{H}$. Multiplied by $r^{c}$, the series converges absolutely and uniformly on strips of bounded width parallel to the ray $\{(r, \theta) \in \mathbb{H}: r>0, \theta=0\}$. 
Proof Since by Proposition 1 the Mellin-Bernstein space is invariant under Mellin translations, the formula of Theorem 6 applies to

$$
g:(r, \theta) \longmapsto t^{c} f(t r, \theta), \quad t>0 .
$$

Rewritten in terms of $f$, it takes the form

$$
\begin{aligned}
r^{c} f(t r, 0)= & \sin (T \log r)\left[\frac{\left(\widetilde{\Theta}_{c} f\right)(t, 0)}{T}+\frac{f(t, 0)}{T \log r}\right. \\
& \left.+T \log r \sum_{k \in \mathbb{Z} \backslash\{0\}} \frac{(-1)^{k+1} e^{k \pi c / T} f\left(t e^{k \pi / T}, 0\right)}{k \pi(k \pi-T \log r)}\right] .
\end{aligned}
$$

Now we substitute $r=e^{ \pm \pi /(2 T)}$ and obtain the two equations

$$
\begin{aligned}
e^{\pi c /(2 T)} f\left(t e^{\pi /(2 T)}, 0\right)= & \frac{\left(\widetilde{\Theta}_{c} f\right)(t, 0)}{T}+\frac{2}{\pi} f(t, 0) \\
& +\frac{1}{2 \pi} \sum_{k \in \mathbb{Z} \backslash\{0\}} \frac{(-1)^{k+1} e^{k \pi c / T} f\left(t e^{k \pi / T}, 0\right)}{k\left(k-\frac{1}{2}\right)}
\end{aligned}
$$

and

$$
\begin{aligned}
e^{-\pi c /(2 T)} f\left(t e^{-\pi /(2 T)}, 0\right)= & -\frac{(\widetilde{\Theta} c f)(t, 0)}{T}+\frac{2}{\pi} f(t, 0) \\
& +\frac{1}{2 \pi} \sum_{k \in \mathbb{Z} \backslash\{0\}} \frac{(-1)^{k+1} e^{k \pi c / T} f\left(t e^{k \pi / T}, 0\right)}{k\left(k+\frac{1}{2}\right)} .
\end{aligned}
$$

Next, by subtraction of these equations, we find that

$$
\begin{aligned}
\left(\widetilde{\Theta}_{c} f\right)(t, 0)= & \frac{T}{2}\left[e^{\pi c /(2 T)} f\left(t e^{\pi /(2 T)}, 0\right)-e^{-\pi c /(2 T)} f\left(t e^{-\pi /(2 T)}, 0\right)\right] \\
& +\frac{T}{\pi} \sum_{k \in \mathbb{Z} \backslash\{0\}} \frac{(-1)^{k} e^{k \pi c / T} f\left(t e^{k \pi / T}, 0\right)}{k\left(4 k^{2}-1\right)} .
\end{aligned}
$$

Finally, employing Proposition 3, we can transform the argument of the polar Mellin derivative from $(t, 0)$ to an arbitrary point $(t, \alpha) \in \mathbb{H}$. This leads us to

$$
\begin{aligned}
\left(\widetilde{\Theta}_{c} f\right)(t, \alpha)= & \frac{T}{2}\left[e^{\pi c /(2 T)} f\left(t e^{\pi /(2 T)}, \alpha\right)-e^{-\pi c /(2 T)} f\left(t e^{-\pi /(2 T)}, \alpha\right)\right] \\
& +\frac{T}{\pi} \sum_{k \in \mathbb{Z} \backslash\{0\}} \frac{(-1)^{k} e^{k \pi c / T} f\left(t e^{k \pi / T}, \alpha\right)}{k\left(4 k^{2}-1\right)},
\end{aligned}
$$

which is the formula of the corollary with $(r, \theta)$ replaced by $(t, \alpha)$. Recalling property (iii) in the definition of $\mathscr{B}_{c, T}^{\infty}$, we easily verify the statement on convergence. 
Remark 4 In order to write the differentiation formula of Corollary 3 in a simpler and more suggestive way, we introduce the central Mellin difference $\delta_{c, h}$ with increment $h>0$ by

$$
\left(\delta_{c, h} f\right)(r, \theta):=h^{c} f(h r, \theta)-h^{-c} f\left(h^{-1} r, \theta\right) .
$$

Note that the right-hand side is a difference of Mellin translations (see [1, p. 2760]) with respect to the first variable of $f$. Then, setting $h:=e^{\pi / T}$, we may write the formula of Corollary 3 as

$$
\left(\widetilde{\Theta}_{c} f\right)(r, \theta)=T\left[\frac{1}{2}\left(\delta_{c, h^{1 / 2}} f\right)(r, \theta)+\frac{1}{\pi} \sum_{k=1}^{\infty} \frac{(-1)^{k}}{k\left(4 k^{2}-1\right)}\left(\delta_{c, h^{k}} f\right)(r, \theta)\right] .
$$

Likewise the Mellin analogue of Boas' differentiation formula may be expressed as

$$
\left(\widetilde{\Theta}_{c} f\right)(r, \theta)=\frac{4 T}{\pi^{2}} \sum_{k=0}^{\infty} \frac{(-1)^{k}}{(2 k+1)^{2}}\left(\delta_{c, h^{k+1 / 2}} f\right)(r, \theta)
$$

where again $h=e^{\pi / T}$. We observe that the summation in (16) has coefficients decaying like $\mathcal{O}\left(k^{-3}\right)$ as $k \rightarrow \infty$ while those of (17) decay like $\mathcal{O}\left(k^{-2}\right)$ only.

Remark 5 For sake of completeness, let us have a quick look at the classical Fourier case. For $f$ belonging to the (Fourier) Bernstein space $B_{w}^{\infty}$, Valiron's sampling formula states that

$$
f(t)=\sin (w t)\left[\frac{f^{\prime}(0)}{w}+\frac{f(0)}{w t}+w t \sum_{k \in \mathbb{Z} \backslash\{0\}} \frac{(-1)^{k} f\left(\frac{k \pi}{w}\right)}{k \pi(w t-k \pi)}\right]
$$

for $t \in \mathbb{R}$. Now we may imitate the proof of Corollary 3 in a Fourier version, that is, we apply this formula to $f(\cdot+x)$, then we substitute $t= \pm \pi /(2 w)$ and subtract the two resulting equations. This way we arrive at the new differentiation formula

$$
\begin{aligned}
f^{\prime}(x)= & \frac{w}{2}\left[f\left(x+\frac{\pi}{2 w}\right)-f\left(x-\frac{\pi}{2 w}\right)\right]+\frac{w}{\pi} \sum_{k \in \mathbb{Z} \backslash\{0\}} \frac{(-1)^{k} f\left(x+\frac{k \pi}{w}\right)}{k\left(4 k^{2}-1\right)} \\
= & \frac{w}{2}\left[f\left(x+\frac{\pi}{2 w}\right)-f\left(x-\frac{\pi}{2 w}\right)\right] \\
& +\frac{w}{\pi} \sum_{k=1}^{\infty} \frac{(-1)^{k}}{k\left(4 k^{2}-1\right)}\left[f\left(x+\frac{k \pi}{w}\right)-f\left(x-\frac{k \pi}{w}\right)\right] .
\end{aligned}
$$

Here the derivative is represented by an alternating series of central differences. Of course, in this formula we can replace the real variable $x$ also by the complex variable $z$. 
We conclude the section with an interesting consequence of Theorem 6, which provides an identity theorem in Mellin-Bernstein spaces.

Corollary 4 Let $f \in \mathscr{B}_{c, T}^{\infty}$, where $c \in \mathbb{R}$ and $T>0$. If $f$ vanishes on the set $\left\{\left(e^{k \pi / T}, 0\right): k \in \mathbb{Z}\right\}$, then

$$
f(r, \theta) \equiv a\left(r e^{i \theta}\right)^{-c} \sin (T(\log r+i \theta))
$$

for some $a \in \mathbb{C}$.

Proof It follows from Theorem 6 that

$$
f(r, 0)=a r^{-c} \sin (T \log r)
$$

for all $r>0$, where $a=\left(\widetilde{\Theta}_{c} f\right)(1,0) / T$.

Now consider the function $g: \mathbb{H} \rightarrow \mathbb{C}$ defined by

$$
g(r, \theta)=a\left(r e^{i \theta}\right)^{-c} \sin (T(\log r+i \theta)) .
$$

Obviously $g$ belongs to $\mathscr{B}_{c, T}^{\infty}$ and $g(r, 0)=f(r, 0)$ for all $r>0$. Thus $\varphi:=f-g$ also belongs to $\mathscr{B}_{c, T}^{\infty}$ and $\varphi(r, 0)=0$ for all $r>0$. By Theorem 2 , it follows that $\varphi(r, \theta) \equiv 0$ and so $f(r, \theta) \equiv g(r, \theta)$ on $\mathbb{H}$.

As a consequence of Corollary 4 , we obtain the following result:

Corollary 5 Let $f \in \mathscr{B}_{c, T}^{p}$, where $c \in \mathbb{R}, T>0$ and $p \in[1, \infty$ [. If $f$ vanishes on the $\operatorname{set}\left\{\left(e^{k \pi / T}, 0\right): k \in \mathbb{Z}\right\}$, then $f$ is identically zero.

Proof Since $\mathscr{B}_{c, T}^{p} \subset \mathscr{B}_{c, T}^{\infty}$, Corollary 4 applies to $f$ and yields that

$$
f(r, \theta) \equiv a\left(r e^{i \theta}\right)^{-c} \sin (T(\log r+i \theta))
$$

for some $a \in \mathbb{C}$. The membership of $f$ in $\mathscr{B}_{c, T}^{p}$ requires that $f(\cdot, 0) \in X_{c}^{p}$. This is possible if and only if $a=0$.

\section{Derivative Sampling in the Mellin Setting}

In this section, we establish an exponential sampling formula for Mellin-bandlimited functions that involves samples of Mellin derivatives up to order $m$. For its proof we shall employ the Paley-Wiener theorem in the Mellin setting in order to profit from the theory of polar-analytic functions.

Theorem 7 Let $f \in B_{c, T}^{2}$ with $c \in \mathbb{R}$ and $T>0$. Then for $m \in \mathbb{N}_{0}:=\mathbb{N} \cup\{0\}$ and $t \in \mathbb{R}^{+}$, there holds the representation

$$
f(t)=t^{-c} \sum_{k \in \mathbb{Z}} e^{c(m+1) k \pi / T} \sin ^{m+1}\left(\log t^{T /(m+1)}\right) \frac{\left(\Theta_{c(m+1) / T}^{m} L_{k, t}\right)\left(e^{k \pi}\right)}{m !}
$$


where

$$
L_{k, t}(r):=\frac{(-1)^{(m+1) k} f\left(r^{(m+1) / T}\right)}{\log \left(t^{T /(m+1)} r^{-1}\right) \operatorname{sinc}^{m+1}\left(\log \left(e^{-k} r^{1 / \pi}\right)\right)} .
$$

The series converges uniformly on compact subsets of $\mathbb{R}^{+}$.

\section{Remark 6}

(i) In response to a question asked by one of the reviewers, we mention that uniform convergence cannot be guaranteed on an interval $] 0, a\left[\subset \mathbb{R}^{+}\right.$even if it is of finite length. This is a peculiarity of Mellin analysis.

(ii) It can be shown that

$$
\left(\Theta_{c(m+1) / T}^{m} L_{k, t}\right)\left(e^{k \pi}\right)=\sum_{j=0}^{m}\left(\Theta_{c}^{j} f\right)\left(e^{(m+1) k \pi / T}\right) \varphi_{m, k, j, T}(t),
$$

where $\varphi_{m, k, j, T}$ are functions on $\mathbb{R}^{+}$depending on $m, k, j$ and $T$ but not on $f$. Thus the sampling formula of Theorem 7 involves samples of the functions $f, \Theta_{c} f, \ldots, \Theta_{c}^{m} f$. The benefit of choosing $m>0$ is that the sample points $e^{(m+1) k \pi / T}(k \in \mathbb{Z})$ are less dense as $m$ increases.

Proof of Theorem 7 For simplicity, we first suppose that $T=m+1$, and so $f \in B_{c, m+1}^{2}$. By the Mellin version of the Paley-Wiener theorem (see Sect. 4 and [1, Thm. 5.1]), $f$ is the restriction to $\mathbb{R}^{+}$of a function $\widetilde{f} \in \mathscr{B}_{c, m+1}^{2}$.

Now let $\mathcal{L}$ be a compact subset of $\mathbb{R}^{+}$. For $t \in \mathcal{L}$, consider the function

$$
F_{t}:(r, \theta) \longmapsto \frac{\tilde{f}(r, \theta)}{\left(\log \left(\frac{r}{t}\right)+i \theta\right) \sin ^{m+1}(\log r+i \theta)} .
$$

It is polar-analytic on $\mathbb{H}$ except for logarithmic poles at the point $(t, 0)$ and the points $\left(r_{k}, 0\right)$, where $r_{k}:=e^{k \pi}$ with $k \in \mathbb{Z}$. Assuming for the moment that

$$
t \in \mathcal{L} \backslash\left\{r_{k}: k \in \mathbb{Z}\right\}
$$

we see that the logarithmic pole at $(t, 0)$ is of order at most 1 and those at $\left(r_{k}, 0\right)$ are of order at most $m+1$.

Next let $\lambda>1$ be a real number and let $K$ and $N$ be integers at least as large as $\lambda^{3 / 2}$. Denote by $\partial \mathcal{R}_{\lambda}$ the positively oriented rectangle with vertices at $\left(e^{-(K+1 / 2) \pi}, \pm \lambda \pi\right)$ and $\left(e^{(N+1 / 2) \pi}, \pm \lambda \pi\right)$. We choose $\lambda$ so large that $\mathcal{L}$ is located inside $\partial \mathcal{R}_{\lambda}$. Then, by the residue theorem for polar-analytic functions (see Theorem 4), we have

$$
I_{\lambda}(t):=\frac{1}{2 \pi i} \int_{\partial \mathcal{R}_{\lambda}}\left(r e^{i \theta}\right)^{c-1} F_{t}(r, \theta) e^{i \theta}(d r+i r d \theta)
$$




$$
=\left(\operatorname{res}_{c} F_{t}\right)(t, 0)+\sum_{k=-K}^{N}\left(\operatorname{res}_{c} F_{t}\right)\left(r_{k}, 0\right) .
$$

Under our restrictions on $t$, we see that

$$
\left(\operatorname{res}_{c} F_{t}\right)(t, 0)=\frac{t^{c} f(t)}{\sin ^{m+1}(\log t)} .
$$

For the calculation of the $c$-residues at $\left(r_{k}, 0\right)$, we use again the factorization (14). It provides us the representation

$$
F_{t}(r, 0)=\frac{H_{k, t}(r)}{(\log r-k \pi)^{m+1}}
$$

with

$$
H_{k, t}(r):=\frac{(-1)^{(m+1) k} f(r)}{\log \left(\frac{r}{t}\right) \operatorname{sinc}^{m+1}\left(\log \left(e^{-k} r^{1 / \pi}\right)\right)} .
$$

In view of Remarks 1 and 2, we find that

$$
\left(\operatorname{res}_{c} F_{t}\right)\left(r_{k}, 0\right)=r_{k}^{c} \frac{\left(\Theta_{c}^{m} H_{k, t}\right)\left(r_{k}\right)}{m !} .
$$

Substituting (19) and (20) in (18) and introducing

$$
A_{m, k}(t):=-\left(\frac{r_{k}}{t}\right)^{c} \sin ^{m+1}(\log t) \frac{\left(\Theta_{c}^{m} H_{k, t}\right)\left(r_{k}\right)}{m !},
$$

we may rewrite (18) as

$$
f(t)-\sum_{k=-K}^{N} A_{m, k}(t)=t^{-c} \sin ^{m+1}(\log t) I_{\lambda}(t) .
$$

So far, in this formula we have excluded those $t \in \mathcal{L}$ with $t=r_{j}$ for some integer $j \in[-K, N]$. Now consider the limit $t \rightarrow r_{j}$ in (22). Obviously, for $j \neq k$ the logarithmic pole of $F_{t}$ at $\left(r_{k}, 0\right)$ does not change its order as $t$ approaches $r_{j}$. Therefore (20) and (21) show that $\lim _{t \rightarrow r_{j}} A_{m, k}(t)=0$ for $j \neq k$. Clearly,

$$
\lim _{t \rightarrow r_{j}} t^{-c} \sin ^{m+1}(\log t) I_{\lambda}(t)=0
$$

for all $r_{j} \in \mathcal{L}$. Hence (22) implies that $\lim _{t \rightarrow r_{j}} A_{m, j}(t)=f\left(r_{j}\right)$. Thus, defining at the exceptional points

$$
A_{m, k}\left(r_{j}\right)=f\left(r_{j}\right) \delta_{k, j}
$$


with Kronecker's delta, we obtain a continuous continuation of the functions $A_{m, k}$ for $k \in \mathbb{Z}$ such that (22) is valid for all $t \in \mathcal{L}$.

Next we want to study the integral $I_{\lambda}(t)$ as $\lambda \rightarrow \infty$. For this, we split it into four parts

$$
I_{\lambda}(t)=I_{\mathrm{hor}}^{-}+I_{\mathrm{vert}}^{\mathrm{r}}+I_{\mathrm{hor}}^{+}+I_{\mathrm{vert}}^{\ell}
$$

corresponding to the lower horizontal, right vertical, upper horizontal and left vertical line segments of $\partial \mathcal{R}_{\lambda}$.

It can be verified that on $\partial \mathcal{R}_{\lambda}$ the estimate (11) is valid. Therefore

$$
\left|I_{\text {hor }}^{ \pm}\right| \leq \frac{3^{m+1}}{2 \pi e^{(m+1) \lambda \pi}} \int_{e^{-(K+1 / 2) \pi}}^{e^{(N+1 / 2) \pi}} \frac{r^{c}|\tilde{f}(r, \pm \lambda \pi)|}{\sqrt{\log ^{2}\left(\frac{r}{t}\right)+\lambda^{2} \pi^{2}}} \frac{d r}{r}
$$

Employing the Cauchy-Schwarz inequality on the right-hand side, we deduce that

$$
\left|I_{\text {hor }}^{ \pm}\right| \leq \frac{3^{m+1}}{2 \pi e^{(m+1) \lambda \pi}}\|\tilde{f}(\cdot, \pm \lambda \pi)\|_{X_{c}^{2}}\left(\int_{0}^{\infty} \frac{1}{\log ^{2}\left(\frac{r}{t}\right)+\lambda^{2} \pi^{2}} \frac{d r}{r}\right)^{1 / 2}
$$

By [1, Thm. 4.2 (i)], we have

$$
\|\tilde{f}(\cdot, \pm \lambda \pi)\|_{X_{c}^{2}} \leq e^{(m+1) \lambda \pi}\|f\|_{X_{c}^{2}}
$$

Furthermore, by an appropriate substitution, we see that

$$
\int_{0}^{\infty} \frac{1}{\log ^{2}\left(\frac{r}{t}\right)+\lambda^{2} \pi^{2}} \frac{d r}{r}=\frac{1}{\lambda \pi} \int_{-\infty}^{\infty} \frac{d x}{x^{2}+1}=\frac{1}{\lambda} .
$$

Thus

$$
\left|I_{\text {hor }}^{ \pm}\right| \leq \frac{3^{m+1}}{2 \pi \sqrt{\lambda}}\|f\|_{X_{c}^{2}}
$$

which shows that $I_{\text {hor }}^{ \pm} \rightarrow 0$ as $\lambda \rightarrow \infty$ uniformly with respect to $t$.

It remains to estimate the contributions from the vertical line segments. Using property (iii) of Mellin-Bernstein spaces (see [1, Def. 4.1]) in conjunction with (11), we find that for some constant $C_{\tilde{f}}>0$, we have

$$
\begin{aligned}
& \left|I_{\mathrm{vert}}^{\mathrm{r}}\right| \leq \frac{3^{m+1} C_{\tilde{f}}}{2 \pi} \int_{-\lambda \pi}^{\lambda \pi} \frac{d \theta}{\sqrt{\left(\left(N+\frac{1}{2}\right) \pi-\log t\right)^{2}+\theta^{2}}} \\
& \leq \frac{3^{m+1} \lambda C_{\tilde{f}}}{\left|\left(N+\frac{1}{2}\right) \pi-\log t\right|} \leq \frac{3^{m+1} \lambda C_{\tilde{f}}}{\left|\left(\lambda^{3 / 2}+\frac{1}{2}\right) \pi-\log t\right|} \longrightarrow 0 \quad \text { as } \lambda \rightarrow \infty
\end{aligned}
$$


uniformly with respect to $t \in \mathcal{L}$. By an analogous consideration, the same result is obtained for $I_{\mathrm{vert}}^{\ell}$.

Altogether we have proved that

$$
f(t)=\sum_{k \in \mathbb{Z}} A_{m, k}(t)
$$

for $f \in B_{c, m+1}^{2}$ and $t \in \mathbb{R}^{+}$with $A_{m . k}$ given by (21). The series converges uniformly on compact subsets of $\mathbb{R}^{+}$.

When $f \in B_{c, T}^{2}$, we consider $g: r \mapsto f\left(r^{(m+1) / T}\right)$ and note that $g \in$ $B_{c(m+1) / T, m+1}^{2}$. Therefore formula (24) applies to $g$ if the role of $c$ is taken by $c(m+1) / T$. Finally, expressing $g$ in terms of $f$ and replacing $t$ with $t^{T /(m+1)}$, we arrive at the desired result.

Let us now consider the cases $m=0$ and $m=1$. For $m=0$ and $f \in B_{c, T}^{2}$ the formula of Theorem 7 takes the form

$$
\begin{aligned}
f(t) & =t^{-c} \sum_{k \in \mathbb{Z}} f\left(e^{k \pi / T}\right) e^{c k \pi / T} \operatorname{sinc}\left(\log \left(e^{-k} t^{T / \pi}\right)\right) \\
& =\sum_{k \in \mathbb{Z}} f\left(e^{k \pi / T}\right) \operatorname{lin}_{c \pi / T}\left(e^{-k} t^{T / \pi}\right), \quad t \in \mathbb{R}^{+} .
\end{aligned}
$$

This result was obtained by a different approach in [9, Thm. 5.2] for $f \in B_{c, \pi T}^{2}$, which allows us to factor out $\pi$ in the resulting series. These authors showed in addition that, when multiplied by $t^{c}$, the series converges uniformly on the whole of $\mathbb{R}^{+}$.

For $m=1$ the formula of Theorem 7 reads as

$$
f(t)=t^{-c} \sum_{k \in \mathbb{Z}} e^{c 2 k \pi / T} \sin ^{2}\left(\log t^{T / 2}\right)\left(\Theta_{2 c / T} L_{k, t}\right)\left(e^{k \pi}\right) .
$$

By the definition of the Mellin derivative, we have

$$
\left(\Theta_{2 c / T} L_{k, t}\right)\left(e^{k \pi}\right)=e^{k \pi} L_{k, t}^{\prime}\left(e^{k \pi}\right)+\frac{2 c}{T} L_{k, t}\left(e^{k \pi}\right) .
$$

A straightforward calculation yields

$$
e^{k \pi} L_{k, t}^{\prime}\left(e^{k \pi}\right)=\frac{f\left(e^{2 k \pi / T}\right)}{\log ^{2}\left(e^{-k \pi} t^{T / 2}\right)}+\frac{2}{T} \frac{e^{2 k \pi / T} f^{\prime}\left(e^{2 k \pi / T}\right)}{\log \left(e^{-k \pi} t^{T / 2}\right)} .
$$

Adding $(2 c / T) L_{k, t}\left(e^{k \pi}\right)$ on both sides, we obtain

$$
\left(\Theta_{2 c / T} L_{k, t}\right)\left(e^{k \pi}\right)=\frac{f\left(e^{2 k \pi / T}\right)}{\log ^{2}\left(e^{-k \pi} t^{T / 2}\right)}+\frac{2}{T} \frac{\left(\Theta_{c} f\right)\left(e^{2 k \pi / T}\right)}{\log \left(e^{-k \pi} t^{T / 2}\right)} .
$$


When we substitute this in (25) and note that

$$
\sin \left(\log t^{T / 2}\right)=(-1)^{k} \sin \left(\log \left(e^{-k \pi} t^{T / 2}\right)\right),
$$

we can build lin functions and arrive at the following result.

Corollary 6 Let $f \in B_{c, T}^{2}$ with $c \in \mathbb{R}$ and $T>0$. Then

$$
\begin{aligned}
f(t)= & \sum_{k \in \mathbb{Z}}\left[f\left(e^{2 k \pi / T}\right) \operatorname{lin}_{c \pi / T}^{2}\left(e^{-k} t^{T /(2 \pi)}\right)\right. \\
& \left.+\frac{2(-1)^{k}}{T}\left(\Theta_{c} f\right)\left(e^{2 k \pi / T}\right) \sin \left(\log t^{T / 2}\right) \operatorname{lin}_{2 c \pi / T}\left(e^{-k} t^{T /(2 \pi)}\right)\right]
\end{aligned}
$$

for $t \in \mathbb{R}^{+}$. The series converges uniformly on compact subsets.

\section{Computational Aspects}

For practical use of formulae (16) and (17) or the sampling formula of Theorem 7 we have to truncate the series. In this section, we want to estimate the truncation errors.

\subsection{The Differentiation Formulae}

As an approximation of the polar Mellin derivative by $2 n$ samples, we have by (16)

$$
\left(\widetilde{\Theta}_{c} f\right)(r, \theta)=T\left[\frac{1}{2}\left(\delta_{c, h^{1 / 2}} f\right)(r, \theta)+\frac{1}{\pi} \sum_{k=1}^{n-1} \frac{(-1)^{k}}{k\left(4 k^{2}-1\right)}\left(\delta_{c, h^{k}} f\right)(r, \theta)\right]+E_{1, n}(f)
$$

and by (17)

$$
\left(\widetilde{\Theta}_{c} f\right)(r, \theta)=\frac{4 T}{\pi^{2}} \sum_{k=0}^{n-1} \frac{(-1)^{k}}{(2 k+1)^{2}}\left(\delta_{c, h^{k+1 / 2}} f\right)(r, \theta)+E_{2, n}(f)
$$

with error terms $E_{1, n}(f)$ and $E_{2, n}(f)$, respectively. Employing property (iii) of the Mellin-Bernstein space, we see that

$$
\left|\left(\delta_{c, t} f\right)(r, \theta)\right| \leq 2 C_{f} r^{-c} e^{T|\theta|}
$$

for any increment $t>0$. Therefore we can estimate the errors as

$$
\left|E_{1, n}(f)\right| \leq \frac{2 C_{f}}{\pi} r^{-c} T e^{T|\theta|} \sum_{k=n}^{\infty} \frac{1}{k\left(4 k^{2}-1\right)}
$$


and

$$
\left|E_{2, n}(f)\right| \leq \frac{8 C_{f}}{\pi^{2}} r^{-c} T e^{T|\theta|} \sum_{k=n}^{\infty} \frac{1}{(2 k+1)^{2}}
$$

For estimating the series on the right-hand side, we may use integral comparison and obtain:

$$
\begin{aligned}
\sum_{k=n}^{\infty} \frac{1}{k\left(4 k^{2}-1\right)} & \leq \int_{n-1}^{\infty} \frac{d x}{x\left(4 x^{2}-1\right)} \\
& =-\frac{1}{2} \log \left(1-\frac{1}{4(n-1)^{2}}\right) \\
& =\frac{1}{2} \sum_{j=1}^{\infty} \frac{1}{j\left[4(n-1)^{2}\right]^{j}} \\
& \leq \frac{1}{2} \sum_{j=1}^{\infty} \frac{1}{\left[4(n-1)^{2}\right]^{j}}=\frac{1}{8(n-1)^{2}-2}
\end{aligned}
$$

and

$$
\sum_{k=n}^{\infty} \frac{1}{(2 k+1)^{2}} \leq \int_{n-1}^{\infty} \frac{d x}{(2 x+1)^{2}}=\frac{1}{4 n-2}
$$

Thus we arrive at the error bounds

$$
\left|E_{1, n}(f)\right| \leq \frac{C_{f} r^{-c} T e^{T|\theta|}}{\pi\left[4(n-1)^{2}-1\right]}
$$

and

$$
\left|E_{2, n}(f)\right| \leq \frac{4 C_{f} r^{-c} T e^{T|\theta|}}{\pi^{2}(2 n-1)} .
$$

\subsection{The Sampling Formula of Theorem 7}

For $f \in B_{c, m+1}^{2}$, the right-hand side of (22) can be seen as a representation of the truncation error. For symmetric truncation, we take $K=N \in \mathbb{N}$ and may choose $\lambda=N^{2 / 3}$. We have to estimate

$$
f(t)-\sum_{k=-N}^{N} A_{m, k}(t)=t^{-c} \sin ^{m+1}(\log t) I_{\lambda}(t) .
$$


Splitting $I_{\lambda}(t)$ as in (23), we deduce from the proof of Theorem 7 that

$$
\left|I_{\text {hor }}^{+}\right|+\left|I_{\text {hor }}^{-}\right| \leq \frac{3^{m+1}}{\pi N^{1 / 3}}\|f\|_{X_{c}^{2}}
$$

and

$$
\left|I_{\text {vert }}^{r}\right|+\left|I_{\text {vert }}^{\ell}\right| \leq \frac{2 \cdot 3^{m+1} N^{2 / 3} C_{\widetilde{f}}}{\left|\left(N+\frac{1}{2}\right) \pi-\log t\right|} .
$$

The proof of [1, Thm. 5.1, p. 9] reveals that we may take

$$
C_{\tilde{f}}=\frac{1}{2 \pi} \int_{-(m+1)}^{m+1}\left|[f]_{M_{c}^{2}}^{\wedge}(c+i t)\right| d t
$$

Using the Cauchy-Schwarz inequality and a relation between the $X_{c}^{2}$-norm of a function $f$ and the $L^{2}$-norm of its Mellin transform (see [9, Lem. 2.6]), we find that

$$
C_{\tilde{f}} \leq \frac{1}{\pi} \sqrt{\frac{m+1}{2}}\left(\int_{-\infty}^{\infty}\left|[f]_{M_{c}^{2}}^{\wedge}(c+i t)\right|^{2} d t\right)^{1 / 2}=\sqrt{\frac{m+1}{\pi}}\|f\|_{X_{c}^{2}} .
$$

Substituting this in (29) and then adding (28) and (29), we obtain

$$
\left|I_{\lambda}(t)\right| \leq \frac{3^{m+1}\|f\|_{X_{c}^{2}}}{\pi N^{1 / 3}}\left(1+\sqrt{\frac{m+1}{\pi}} \frac{2 N}{\left|\left(N+\frac{1}{2}\right)-\log \left(t^{1 / \pi}\right)\right|}\right) .
$$

Thus,

$$
\begin{aligned}
\left|f(t)-\sum_{k=-N}^{N} A_{m, k}(t)\right| \leq & t^{-c}\left|\sin ^{m+1}(\log t)\right| \frac{3^{m+1}\|f\|_{X_{c}^{2}}}{\pi N^{1 / 3}} \\
& \times\left(1+2 \sqrt{\frac{m+1}{\pi}}+o(1)\right) \quad \text { as } N \rightarrow \infty
\end{aligned}
$$

holding for $f \in B_{c, m+1}^{2}$.

By a transformation as at the end of the proof of Theorem 7, we can deduce a corresponding estimate for $f \in B_{c, T}^{2}$ with an arbitrary $T>0$. Indeed, defining

$$
E_{N}[f](t):=f(t)-t^{-c} \sum_{k=-N}^{N} e^{c(m+1) k \pi / T} \sin ^{m+1}\left(\log t^{T /(m+1)}\right) \frac{\left(\Theta_{c(m+1) / T}^{m} L_{k, t}\right)\left(e^{k \pi}\right)}{m !},
$$

we find that

$$
\left|E_{N}[f](t)\right| \leq t^{-c}\left|\sin ^{m+1}\left(\log t^{T /(m+1)}\right)\right| \sqrt{\frac{T}{m+1}} \frac{3^{m+1}\|f\|_{X_{c}^{2}}}{\pi N^{1 / 3}}
$$




$$
\times\left(1+2 \sqrt{\frac{m+1}{\pi}}+o(1)\right) \quad \text { as } N \rightarrow \infty .
$$

Acknowledgements Open Access funding provided by Projekt DEAL. The authors want to thank the reviewers for their careful reading and evaluation of the present paper.

\section{Compliance with Ethical Standards}

Conflicts of interest The authors declare that they have no conflict of interest.

Open Access This article is licensed under a Creative Commons Attribution 4.0 International License, which permits use, sharing, adaptation, distribution and reproduction in any medium or format, as long as you give appropriate credit to the original author(s) and the source, provide a link to the Creative Commons licence, and indicate if changes were made. The images or other third party material in this article are included in the article's Creative Commons licence, unless indicated otherwise in a credit line to the material. If material is not included in the article's Creative Commons licence and your intended use is not permitted by statutory regulation or exceeds the permitted use, you will need to obtain permission directly from the copyright holder. To view a copy of this licence, visit http://creativecommons.org/licenses/by/4.0/.

\section{References}

1. Bardaro, C., Butzer, P.L., Mantellini, I., Schmeisser, G.: A fresh approach to the Paley-Wiener theorem for Mellin transforms and the Mellin-Hardy spaces. Math. Nachr. 290, 2759-2774 (2017)

2. Bardaro, C., Butzer, P.L., Mantellini, I., Schmeisser, G.: Quadrature formulae for the positive real axis in the setting of Mellin analysis: sharp error estimates in terms of the Mellin distance. Calcolo 55(3), article 26 (2018)

3. Bardaro, C., Butzer, P.L., Mantellini, I., Schmeisser, G.: Development of a new concept of polar analytic functions useful in Mellin analysis. Complex Var. Elliptic Equ. 64(12), 2040-2062 (2019)

4. Bardaro, C., Butzer, P.L., Mantellini, I., Schmeisser, G.: Integration of polar-analytic functions and applications to Boas' differentiation formula and Bernstein's inequality in Mellin frame. Boll. Unione Mat. It. https://doi.org/10.1007/s40574-020-00226-9 (published open access online: 28 May 2020)

5. Brown, J.W., Churchill, R.V.: Complex Variables and Applications, 8th edn. McGraw-Hill, New York (2009)

6. Butzer, P.L., Berens, H.: Semigroups of Operators and Approximation. Springer, Berlin (1967)

7. Butzer, P.L., Jansche, S.: A direct approach to the Mellin transform. J. Fourier Anal. Appl. 3, 325-375 (1997)

8. Butzer, P.L., Jansche, S.: The exponential sampling theorem of signal analysis. Atti Sem. Mat. Fis. Univ. Modena Suppl. 46, 99-122 (1998). (special issue dedicated to Professor Calogero Vinti)

9. Butzer, P.L., Jansche, S.: A self-contained approach to Mellin transform analysis for square integrable functions, applications. Integral Transforms Spec. Funct. 8, 175-198 (1999)

10. Butzer, P.L., Schmeisser, G., Stens, R.L.: Basic relations valid for the Bernstein space $B_{\sigma}^{p}$ and their extensions to functions from larger spaces with error estimates in terms of their distances from $B_{\sigma}^{p}$. J. Fourier Anal. Appl. 19, 333-375 (2013)

11. Higgins, J.R.: Sampling Theory in Fourier and Signal Analysis. Foundations. Oxford University Press, Oxford (1996)

12. Hille, E.V.: Analytic Function Theory, vol. 1. Chelsea Publishers Co., New York (1959)

13. Pesenson, I.Z.: Boas-type formulas and sampling in Banach spaces with applications to analysis on manifolds. In: Zayed, A.I., Schmeisser, G. (eds.) New Perspectives on Approximation and Sampling Theory, pp. 39-61. Springer, Cham (2014)

14. Pesenson, I.Z.: Sampling formulas for groups of operators in Banach spaces. Sampl. Theory Signal Image Process. 14(1), 1-16 (2015)

15. Silverman, H.: Polar form of Cauchy-Riemann equations. Primus 10(3), 241-245 (2000) 
Publisher's Note Springer Nature remains neutral with regard to jurisdictional claims in published maps and institutional affiliations.

\section{Affiliations}

\section{Carlo Bardaro ${ }^{1}$. Paul L. Butzer ${ }^{2}$. Ilaria Mantellini ${ }^{1}$. Gerhard Schmeisser ${ }^{3}$}

Carlo Bardaro

carlo.bardaro@unipg.it

Paul L. Butzer

butzer@rwth-aachen.de

Ilaria Mantellini

mantell@dmi.unipg.it

1 Department of Mathematics and Computer Sciences, University of Perugia, via Vanvitelli 1, 06123 Perugia, Italy

2 Lehrstuhl A für Mathematik, RWTH Aachen, Templergraben 55, 52056 Aachen, Germany

3 Department Mathematik, Friedrich-Alexander University Erlangen-Nürnberg, Cauerstr. 11, 91058 Erlangen, Germany 